\title{
Belief Updating and Paranoia in Individuals with Schizophrenia
}

Julia M Sheffield ${ }^{1}$, Praveen Suthaharan², Pantelis Leptourgos², Philip R Corlett ${ }^{2}$

1, Vanderbilt University Medical Center, Department of Psychiatry \& Behavioral Sciences; 2, Department of Psychiatry, Connecticut Mental Health Center, Yale University, New Haven, United States 


\section{Abstract}

Background and Hypothesis: Persecutory delusions are among the most common delusions in schizophrenia and represent the extreme end of the paranoia continuum. Paranoia is accompanied by significant worry and distress. Identifying cognitive mechanisms underlying paranoia is critical for advancing treatment. We hypothesized that aberrant belief updating, which is related to paranoia in human and animal models, would also contribute to persecutory beliefs in individuals with schizophrenia.

Study Design: Belief updating was assessed in 42 schizophrenia and 44 healthy participants, using a 3-option probabilistic reversal learning (3-PRL) task. Hierarchical Gaussian filter (HGF) was used to estimate computational parameters of belief updating. Paranoia was measured using the Positive and Negative Syndrome Scale (PANSS) and the revised Green et al. Paranoid Thoughts Scale (R-GPTS). Unusual thought content was measured with the Psychosis Symptom Rating Scale (PSYRATS) and the Peters et al. Delusions Inventory (PDI-21). Worry was measured using the Dunn Worry Questionnaire.

Results: Consistent with prior work, paranoia was significantly associated with elevated winswitch rate, prior on volatility and sensitivity to volatility in both schizophrenia and across the whole sample. These relationships were specific to paranoia and did not extend to unusual thought content or measures of anxiety. We did, however, find a significant indirect effect of paranoia on the relationship between prior beliefs about volatility and worry.

Conclusions: This work provides evidence that relationships between belief updating parameters and paranoia extend to schizophrenia, may be specific to persecutory beliefs, and contribute to theoretical models implicating worry in the maintenance of persecutory delusions. 


\section{Introduction}

Persecutory delusions - beliefs that others intend you harm - are among the most common delusions in schizophrenia ${ }^{1,2}$. They exist on a continuum with mild suspicion in the general nonclinical population ${ }^{3}$. Paranoia is related to reduced well-being, violence towards self and others $^{4-6}$, conspiracy theorizing 7 , and less adherence to public health measures ${ }^{8}$. Identifying the underlying mechanisms of paranoia will have clinical as well as broader societal impact.

Worry and negative self-beliefs contribute to paranoia and persecutory beliefs ${ }^{9}$. These can be targeted with psychotherapy, with a large and specific impact on persecution (relative to other delusions) ${ }^{10}$. However, a deeper appreciation of the underlying mechanisms of paranoia is necessary. Belief updating is one candidate ${ }^{11}$. The challenge of believing and updating one's beliefs is to remain robust to real-world variability while simultaneously flexible in the face of true change. Anecdotally, expecting a volatile world licenses worry and paranoia ${ }^{12}$. We sought to test this hypothesis experimentally.

Recent work has linked beliefs about volatility $\left(\mu_{3}^{0}\right)$ and sensitivity to volatility $(\kappa)$ to paranoia in humans and rats administered amphetamine ${ }^{13}$. Furthermore, the increase in uncertainty occasioned by the pandemic increased participants' expectations of change and their paranoia ${ }^{8}$. Heightened sensitivity to volatility shapes one's prior beliefs about the volatility of the environment: the perception that things are changing depicts an unstable environment that is difficult to predict. The contributions of expectations about and learning from volatility to persecutory beliefs in schizophrenia have not yet been established.

Furthermore, belief updating is relevant to and impacted by anxiety. Both trait anxiety and state anxiety are related to compromised adjustment of learning rates in the context of environmental uncertainty ${ }^{14,15}$. Worry in particular is strongly associated with paranoia ${ }^{16,17}$. It keeps threat beliefs in mind, encouraging elaboration and actions (e.g. avoidance) that nourish the persecutory belief ${ }^{18}$. Cognitive-behavioral therapy for worry reduces persecutory delusion severity ${ }^{19}$. Understanding the relationship between belief updating, paranoia, and worry may connect computational psychiatry and the precision it affords with the therapeutic opportunities of $\mathrm{CBT}^{20}$.

We examined: 1) whether the relationship between belief updating and paranoia extends to schizophrenia, 2) the specificity of this relationship as compared with overall unusual thought content and anxiety, and 3) the indirect effect of paranoia on volatility and worry, hypothesizing that: a greater prior on volatility ("the world is frequently changing") contributes to paranoid thinking ("I will be harmed"), which fuels worry. 


\section{Methods}

\section{Participants}

Forty-five (45) individuals with a schizophrenia-spectrum disorder and 46 healthy control participants with no psychiatric history were recruited. Individuals aged 18-55 were identified from the Vanderbilt University Medical Center (VUMC) Psychotic Disorders Program. Diagnoses were confirmed by a Structured Clinical Interview of the DSM-IV-TR (SCID) $)^{21}$ completed by a trained rater and signed off in a consensus meeting. All participants were free of major physical or neurological illness, active substance use disorder, and significant head injury, and had an estimated IQ of $>79$. Healthy controls did not have a first-degree relative with a psychotic disorder or any current psychotropic medication use. One healthy participant was excluded after study completion, due to a current prescription of Adderall. Study protocol was approved by the Vanderbilt Institutional Review Board (IRB) and informed consent was provided by all research participants prior to study participation.

Participants were recruited between December 2020 - October 2021. Given limitations of inperson research participation as a result of the pandemic, the majority of participants (91\%) conducted the study at home on a personal laptop. A smaller proportion of participants (9\%) who did not have access to a laptop completed the study in-person.

\section{Experimental Paradigm}

Belief updating was measured using the 3-option probabilistic reversal learning (3-PRL) task ${ }^{13}$ (Figure 1a). Participants were presented with three decks of cards on a computer screen and told that each deck includes both winning (+100 points) and losing ( -50 points) cards, but that some decks win more often than others. They were instructed to find the 'best' deck, and that the best deck might change, whereupon they should try and find the new best deck. There were 160 trials divided into 4 blocks, with an optional break in between each block.

Initially, the decks yielded $90 \%, 50 \%$, and $10 \%$ reward, for the $1^{\text {st }}$ and $2^{\text {nd }}$ blocks ( 80 trials). Unbeknownst to the participant, these contingencies changed at the start of block 3 to 80-4020. This made it more difficult for the participant to discern whether a loss was due to probabilistic noise or due to the best deck changing ${ }^{13}$.

\section{Delusion Assessments}

In the schizophrenia group, overall delusion severity was assessed using the PSYRATS ${ }^{23}$, an interviewer-rated assessment of the conviction, preoccupation, distress and functional impairment related to a specific delusional belief. Collaboratively with a trained interviewer, schizophrenia participants identified a delusion they were either currently experiencing or (if not experiencing any current delusions) have experienced strongly in the past. This conversation was facilitated by review of previous medical records and past clinical interview from the research database. Once a statement that encapsulated their delusional belief was 
identified it was rated for severity in the past week. Delusional beliefs as rated by the PSYRATS included a variety of themes (e.g. grandiose, religious, guilt, and persecution). Paranoia was measured using the PANSS ${ }^{22}$ Suspiciousness/Persecution (P6) scores, which provided a more specific measure of interview-rater paranoia.

Across all participants, overall delusional ideation was measured using the PDI- $21^{25}$, which assesses endorsement (yes/no), level of distress, conviction, and preoccupation related to a variety of delusional beliefs. Total PDI score was calculated by summing all of the items. Paranoia was assessed using the R-GPTS ${ }^{24}$, which includes a 10 -item scale measuring persecutory ideation (GPTS-b).

\section{Other Symptom Assessments}

Anxiety was assessed using: 1 ) the Beck Anxiety Inventory $\left.(B A I)^{26}, 2\right)$ the Dunn Worry Questionnaire (DWQ $)^{16}$, which includes 10 -items related to how worry interferes with daily life and a perceived sense of lack of control over worry, as well as a 5-item assessment of paranoia worries, 3) the Perseverative Thinking Questionnaire (PTQ) ${ }^{28}$ and 4) the Liebowitz Social Anxiety Scale $\left(\right.$ LSAS) ${ }^{29}$. Depression was assessed using the Beck Depression Inventory (BDI) ${ }^{27}$. Worry was measured through

\section{Behavioral Analysis}

The primary behavioral measure of interest was win-switch rate. Win-switching occurs when an individual chooses an alternative deck even after receiving positive feedback (+100 points). Win-switch rates were calculated as the number of win-switch decisions divided by the number of trials in which they received positive feedback. We also investigated lose-stay decisions, which reflect a decision to persist with a chosen deck after receiving negative feedback (-50 points). Lose-stay rates were calculated as the number of lose-stay decisions divided by the number of trials with negative feedback.

\section{Computational modelling}

Computational modelling was conducted on task data using the Hierarchical Gaussian Filter (HGF) toolbox ${ }^{30,31}$, and MATLAB 2020b (MathWorks, Natick, MA). Belief updating parameters were calculated for the 90-50-10 reward contingencies ( $1^{\text {st }}$ and $2^{\text {nd }}$ blocks) and the 80-40-20 contingencies ( $3^{\text {rd }}$ and $4^{\text {th }}$ blocks). Participant data was entered as separate column vectors for each block, modeling deck choice (deck 1,2, or 3) and outcome (win or loss), using an autoregressive 3-level HGF multi-arm bandit configuration for the perceptual model, paired with a decision model that contains $\mu_{3}^{0}$ (Softmax-mu3 decision model) to model an individual's behavior (stay on the same deck or switch to a different deck) driven by their belief of how volatile the task environment is (Figure 1b). Belief updating trajectories are represented as probability distributions that encode belief content and uncertainty and were specific to each participant, due to the probabilistic and performance-dependent nature of the task. Analyses 
were completed using scripts that have been previously reported and shared ${ }^{8}$ https://github.com/psuthaharan/covid19paranoia.

The HGF estimates terms at multiple levels that describe different aspects of decision-making. Level 1 ( $x 1$ ) characterizes trial-by-trial task feedback (win or loss), Level 2 (x2) explains stimulusoutcome associations (reward probabilities underlying each deck), and Level $3(x 3)$ is the highest level and reflects perception of the task's overall volatility (i.e. how often the 'best deck' changes). The primary computational parameter of interest was $\mu_{3}^{0}$. This parameter estimates the initial (prior) beliefs of the Level 3 (overall) volatility. In other words, it reflects the prior expectation of how volatile the task environment is. Higher $\mu_{3}^{0}$ values indicate a greater prior on volatility (i.e. a stronger expectation that the contingencies will shift erratically). We were also interested in $\kappa$, which captures sensitivity to perceived unexpected (phasic) task volatility (changes), with higher $\kappa$ (sensitivity to volatility) also contributing to more rapid and extensive updating of beliefs.

In addition, $\omega_{2}$ and $\omega_{3}$ were calculated. These parameters reflect the evolution rate of the task environment at the level of contingencies $\left(\omega_{2}\right)$ and volatility $\left(\omega_{3}\right)$, with higher levels implying more rapid change. $\omega_{2}$ is an estimate of expected uncertainty (i.e. estimation of the instability of reward contingencies). Lower $\omega_{2}$ indicates slower adjustment of beliefs about the value of each deck choice. $\omega_{3}$ is 'metavolatility' which indexes how stable the changes in underlying contingencies of the decks might be. Higher values imply a more rapid adjustment of their volatility belief.

Previous reports have tested the propriety of the 3-level HGF through simulations and comparison with alternative models ${ }^{13}$. Simulation has been shown to recover group difference in behavior between high and low paranoia, whereas simpler models (e.g. those that included only a 2-level HGF and weighted-prediction error reinforcement models) did not recapitulate group differences. This suggests appropriateness of the 3-level HGF for modeling belief updating behavior in relationship to paranoia.

\section{Data Analysis}

Participant demographics were compared between groups (Controls, Schizophrenia) using chisquare and independent-samples t-tests (Table 1). Multivariate outliers were identified using Mahalanobis Distance calculations for the R-GPTS total score and the win-switch rate. Four multivariate outliers were identified and excluded from further analysis ( 1 healthy and 3 schizophrenia participants), in addition to the 1 healthy participant who was prescribed a psychotropic medication. The final sample size for all analyses was 42 schizophrenia and 44 healthy participants.

Group differences in behavioral measures (win-switch and lose-stay) and computational parameters $\left(\mu_{3}^{0}, \kappa, \omega_{2}, \omega_{3}\right)$ were tested in two separate multivariate analysis of variance (MANOVA) models. Repeated measures ANOVA was also used to determine whether computational parameters differed based on the reward contingencies (main effect of block) 
and whether there was a significant group by block interaction. In the absence of a group by block interaction, computational parameters averaged across the blocks were used for further analysis.

Relationships between belief updating measures and symptom assessments were analyzed in two-tailed bivariate Pearson's correlations. Hypothesized relationships between paranoia, winswitch, $\mu_{3}^{0}$ and $\kappa$ were examined first. Additional analyses were Bonferroni-corrected. Given similar demographics across groups, no covariates were included in our primary analyses, however results with covariates (age, sex, race) are outlined in the Supplement.

Cross-sectional mediation analysis was conducted using the Process macro (v4.0) in SPSSv28 (model 4). 


\section{Results}

\section{Group Differences in Behavioral Performance}

Individuals with schizophrenia displayed slightly slower reaction time $(F(1,84)=3.77, p=.06)$ and fewer achieved reversals $(F(1,84)=3.35 p=.07)$, although the differences were not significant. They exhibited significantly more win-switch $(F(1,84)=9.21, p=.003)$ and less lose-stay $(F(1,84)=4.53, p=.04)$ behavior than healthy participants, although lose-stay differences did not survive correction ( $p_{\text {critical }}=.03$ ). Schizophrenia participants shifted to a new card deck after a win almost $3 \mathrm{x}$ as often as controls ( $11 \%$ vs. $4 \%$ of the time) (Figure $2 \mathrm{a}$ ).

\section{Relationships Between Behavioral Performance and Paranoia}

In the schizophrenia group, interviewer-rated paranoia (as measured by the PANSS P6) was significantly associated with win-switch $(r(42)=.36, p=.02)$ but not lose-stay rate $(r(42)=-.08$, $\mathrm{p}=.61$ ). Similarly, self-reported paranoia (GPTS-b) was significantly associated with win-switch rate across all participants $(r(86)=.36, p<.001)$ (Figure 3$)$. Lose-stay was not significantly associated with self-reported paranoia $(r(86)=-.01, p=.94)$.

\section{Relationships Between Behavioral Performance and Overall Unusual Thought Content}

In the schizophrenia group, interviewer-rated measures of overall delusion severity (PSYRATS) were not significantly associated with win-switch $(\mathrm{r}(42)=.03, \mathrm{p}=.87)$ or lose-stay behavior $(r(42)=.14, p=.39)$. Similarly, self-reported delusional ideation (PDI-21) was not associated with win-switch $(r(86)=.14, p=.21)$ or lose-stay $(r(86)=.05, p=.66)$ behavior across the whole sample.

\section{Group Differences in Computational Parameters}

Repeated-measures ANOVA did not reveal any significant group by block interactions for computational parameters ( $\left.p^{\prime} s>.25\right)$. Therefore, average parameters were examined for group differences. Parameter estimates were within the expected range, based on prior analyses in large-scale datasets ${ }^{8,32}$. No significant group differences were observed for computational parameters between schizophrenia and healthy participants (Figure 2b).

\section{Belief Updating and Paranoia}

In the schizophrenia group, interviewer-rated paranoia was significantly associated with $\mu_{3}^{0}$ $(\mathrm{r}(42)=.48, \mathrm{p}=.001)$ and $\kappa(\mathrm{r}(42)=.33, \mathrm{p}=.03)$ (Figure 3$)$, but not $\omega_{2}(\mathrm{r}(42)=-.27, \mathrm{p}=.08)$ or $\omega_{3}$ $(r(42)=-.25, p=.10)$.

Across the whole sample, a similar pattern was observed. Greater $\mu_{3}^{0}(\mathrm{r}(86)=.23, \mathrm{p}=.03)$ and $\kappa$ $(r(86)=.25, p=.02)$ were significantly related to paranoia, but $\omega_{2}(r(86)=-.21, p=.06)$ and $\omega_{3}$ $(r(86)=-.17, p=.12)$ were not. 
Computational parameters were not significantly associated with either interview-rated delusion severity (all p's >.08) or self-reported delusional ideation across the whole sample $\left(p^{\prime} s>.35\right)$.

Finally, participants were split into those with elevated and low paranoia, based on their RGPTS-b scores. Detailed in the Supplement (Figure S1), we observed significantly greater $\mu_{3}^{0}$ and $\kappa$ in those with elevated paranoia.

\section{Relationships with anxiety and depression}

Groups did not differ on self-reported anxiety or depression for any measure after correcting for multiple comparisons ( $p^{\prime} s>.15$ ). Interestingly, we also did not observe significant associations between $\mu_{3}^{0}$ or win-switch rate with measures of anxiety, including physiological anxiety symptoms, social anxiety, overall worry, or perseverative thinking (all p's $>.15$ ).

Persecutory worry, as measured by the Dunn Worry Questionnaire, was, however, significantly associated with win-switch behavior $(r(86)=.37, p<.001)$ and $\mu_{3}^{0}(r(86)=.25, p=.02)$. General, nonpersecutory worry as measured on the Dunn Worry Questionnaire was NOT associated with win-switch behavior $(r(86)=.09, p=.42)$ or $\mu_{3}^{0}(r(86)=.05, p=.63)$, again suggesting some specificity to paranoid thinking. $\kappa$ was not significantly associated with general $(r(86)=-.09$, $p=.94)$ or persecutory worry $(r(86)=.09, p=.42)$

\section{Indirect Effect Analysis}

In the schizophrenia sample, paranoia (PANSS P6) demonstrated a significant indirect effect on the relationship between $\mu_{3}^{0}$ and overall worry (DWQ total) (Figure 4a; 95\% Cl [.35, 3.8]), in the context of significant relationships between $\mu_{3}^{0}$ and paranoia $(\mathrm{t}=3.43, \mathrm{p}=.001)$ and paranoia and worry ( $t=2.65, p=.01)$. Similarly, in the whole sample, paranoia (GPTS-b) had a significant indirect effect on the relationship between $\mu_{3}^{0}$ and overall worry (Figure $4 b ; 95 \% \mathrm{Cl}[.07,1.4]$ ), in the context of significant relationships between $\mu_{3}^{0}$ and paranoia $(t=2.18, p=.03)$ and paranoia and worry $(t=3.54, p<.001)$.

\section{Covariate analyses}

Although our groups did not differ on these variables, we explored how controlling for age, sex, and race impacted our primary findings. These are outlined in detail in the Supplement. Briefly, all group differences and relationships with paranoia remained when these covariates were included, except that the relationship between computational parameters $\left(\mu_{3}^{0}\right.$ and $\left.\kappa\right)$ and selfreported paranoia was attenuated $\left(\mu_{3}^{0}: p=.16 ; \kappa: p=.13\right)$. This appeared to be driven by the inclusion of race, as relatively more African-American participants demonstrated elevated paranoia. Similarly, the mediation analyses were significant when controlling for age and sex, but not race. 
Additionally, we investigated the relationship between antipsychotic medication dose and belief updating in our schizophrenia cohort using Chlorpromazine (CPZ)-equivalence estimates. CPZ-equivalence was unrelated to belief updating parameters $\left(\mu_{3}^{0}: p=.91 ; \kappa: p=.72 ; \omega_{2}: p=.97\right.$, $\left.\omega_{3}: p=.95\right)$. Inclusion as a covariate in relationships between belief updating and suspiciousness (P6) revealed significant associations with $\mu_{3}^{0}(r=.37, p=.02)$ and an attenuated relationship with $\kappa(r=.28, p=.07)$. 


\section{Discussion}

The current study replicates prior work connecting aberrant belief updating with paranoia and extending the relationship to individuals with schizophrenia. We replicate elevated win-switch rates in schizophrenia ${ }^{33,34}$, elevated prior on volatility and sensitivity to volatility in high paranoia ${ }^{8,32}$, and relationships between self-reported paranoia and belief updating ${ }^{32}$. We then demonstrate that elevated win-switch behavior, prior on volatility, and sensitivity to volatility are significantly associated with greater persecution beliefs in individuals with schizophrenia, suggesting prior findings in a heterogenous sample do indeed extend along the persecutory delusion continuum. Belief updating parameters may also be specific to paranoid thinking, as they were not significantly associated with overall unusual thought content or measures of anxiety and depression. Further, we demonstrate a novel, significant indirect effect of paranoia on the relationship between volatility and worry.

Belief updating has been increasingly implicated in delusional thinking ${ }^{36}$. It represents a key challenge that the brain faces; learning to predict the environment with sufficient confidence, while remaining flexible enough when confronted with significant change ${ }^{37}$. Our data and models implicate an expectation of change (or prior), and a learning rate, often tethered to a prediction error - we should learn most in situations where prediction errors are large and variable. This may be reflected in mesocortical and mesostriatal dopamine signaling, as well as noradrenergic responses in locus coeruleus, insula and anterior cingulate cortex ${ }^{35}$.

We observed significant elevations in win-switch rate in schizophrenia, but not diagnostic group differences in belief updating parameters. Elevated win-switch rate is frequently observed in schizophrenia ${ }^{36,37}$. Our lack of group differences in computational parameters is in contrast to a recent neuroimaging study of a 2-option PRL task in schizophrenia which found elevated $\mu_{3}^{0}$ and $\kappa$ in stable, medicated patients ${ }^{33}$. Elevated volatility priors in schizophrenia were related to activity in the dorsolateral prefrontal cortex (dIPFC), suggesting involvement of the dIPFC in this experience of volatility during decision-making. Intriguingly, although diagnostic groups did not differ, we replicated prior work showing that individuals with elevated paranoia have elevated $\mu_{3}^{0}$ and $\kappa^{32}$. While the groups were unbalanced and therefore this analysis was largely exploratory, this suggests that elevations in these belief updating parameters may be more related to paranoid beliefs than diagnosis.

One of the main goals of the current study was to determine whether individuals with schizophrenia exhibit the same continuous relationships between paranoia and belief updating as has been observed in the general population, and further determine if these relationships extend to delusional ideation more broadly. We found that elevated prior on volatility $\left(\mu_{3}^{0}\right)$ and sensitivity to volatility $(\kappa)$ were significantly associated with the suspiciousness/persecution rating on the PANSS. In our outpatient sample, item scores ranged from 1 to 5 , with an average score of 3, representing guardedness and a distrustful attitude but not a severe persecutory delusion in most participants. That said, the vast majority of our schizophrenia participants (at least $89 \%$ according to their PSYRATS statement and review of medical records) have experienced persecutory delusions in the past. This suggests that even when persecutory 
delusion severity is relatively low, the prior belief that the world is unpredictable and frequently changing contributes to a paranoid thinking style which may persist even in the absence of an acute delusional state.

Intriguingly, these belief updating measures were not significantly associated with delusional ideation more broadly. The importance of delusional 'themes' has been long-debated in the clinical literature ${ }^{38}$. On the one hand, understanding how an individual develops a false and incorrigible belief is broadly important; yet, conceptualizing the type of delusion may help guide specific treatment recommendations ${ }^{39,40}$. The relationship between belief updating measures and paranoia, but not overall delusion severity, suggests importance of these belief updating parameters for the experience of threat and suspiciousness specifically. It is also important to note, however, that relationships with unusual thought content may be more difficult to pick up using standard assessment tools, particularly in this relatively small and stable sample. Future studies in more acute patients with a wider range of delusional thought content will help adjudicate the question of specificity to delusional theme.

An additional goal of the study was to examine relationships between belief updating, paranoia and anxiety. Trait anxiety is associated with a deficit in adjusting learning rates when environmental volatility changes ${ }^{14}$. State anxiety is related to underestimation of environmental uncertainty, contributing to reduced learning rates ${ }^{15}$. Anxiety, in particular worry, contributes to paranoia and persecutory delusions ${ }^{17,41}$. Interestingly, we found that physiological anxiety, social anxiety, and generalized worry were not significantly associated with belief updating parameters in this sample, including no significant relationships with learning rates $\left(\omega_{2}\right)$. We did, however, observe a significant association between a volatility prior and greater persecutory worry (e.g. "worries about someone trying to harm me have been really hard to control"), but not general worry (e.g. "In my mind I have been going over problems again and again").

The relationship between worry, paranoia and volatility prior was further tested by measuring the indirect effect of paranoia on the relationship between volatility and worry, revealing that a stronger prior on volatility was associated with paranoia, which was in turn associated with general worrying. Theoretical accounts of paranoia have suggested that worrying keeps threat beliefs in mind, elaborates on them and drives behavior ${ }^{42}$. A cognitive-behavioral therapy (CBTp) intervention targeting worry significantly reduced persecutory delusion severity in individuals with schizophrenia, validating it as a contributory causal factor to maintenance of persecutory delusions ${ }^{19}$. It remains unknown, however, what makes someone feel paranoid in the first place and what additional cognitive processes may be at play. These findings are the first to suggest that a prior belief that the world is volatile contributes to paranoid beliefs, which in turn fuel worry. While replication is needed, particularly in a longitudinal cohort and/or intervention study, this implies that a prior on volatility represents a future target for cognitive-behavioral and other psychosocial interventions, and that we might employ this computational marker as a metric of change with therapy or as a prospective harbinger of therapeutic success. 
Related to the notion of psychosocial impact on volatility priors, individuals with high paranoia in our sample were significantly more likely to be African-American. It is critical to note that this dataset is too small to draw conclusions about the influence of race or racial discrimination on paranoia and volatility; however, it opens up an interesting line of inquiry around how these volatility priors develop. Future research should examine whether discrimination, urbanicity, and poverty (known risk factors for the development of psychosis) ${ }^{43}$ influence belief updating.

Limitations of this study include small sample size, relatively low symptom severity of our participants and completion of the study virtually. Small sample size increases the risk of false positives ${ }^{44}$, however we note that the effect size of the group difference in win-switch rate and correlations between belief updating and paranoia are highly similar to those observed in previous studies ${ }^{13}$, including a sample of several hundred individuals ${ }^{8}$, increasing our confidence in these findings. Lower symptom ratings in our schizophrenia participants may have limited our ability to detect relationships with unusual thought content, which is more dependent on the presence of an unusual belief (e.g. "I can read people's minds") as opposed to more common paranoid thoughts (e.g. "certain individuals have had it in for me").

Determining the specificity of these belief updating parameters to paranoia versus delusion severity generally will require replication, ideally in a larger longitudinal sample with a greater range of delusion severity. Finally, due to lockdown conditions, the vast majority of participants completed the study virtually. This may have introduced additional noise into the dataset and slightly limited the ability of the interviewer to fully assess clinical symptom ratings. Reliability of the PANSS over telehealth has not been specifically established, yet work from other fields (autism, dementia) suggest validity of conducting clinical assessments virtually ${ }^{45,46}$. Furthermore, prior work on paranoia and belief updating was replicated in the office and virtually (over MTurk) ${ }^{32}$, again suggesting durability of these relationships regardless of location completed.

In summary, these findings provide additional evidence for a role of belief updating in paranoia (particularly elevated prior on volatility and sensitivity to volatility) and extend these relationships to individuals with schizophrenia. They further suggest specificity of these relationships to paranoia, as opposed to overall delusional ideation and anxiety. Finally, they suggest that a prior belief that the world is volatile and unstable may contribute to paranoid thoughts, driving worry. These findings implicate prior on volatility as a potential target for treatment of persecutory delusions. 


\section{References}

1. Sartorius N, Jablensky A, Korten A, et al. Early manifestations and first-contact incidence of schizophrenia in different cultures: A preliminary report on the initial evaluation phase of the WHO Collaborative Study on Determinants of Outcome of Severe Mental Disorders. Psychol Med. 1986;16(4):909-928. doi:10.1017/S0033291700011910

2. Picardi A, Fonzi L, Pallagrosi M, Gigantesco A, Biondi M. Delusional themes across affective and non-affective psychoses. Front Psychiatry. 2018;9(APR):132. doi:10.3389/FPSYT.2018.00132/BIBTEX

3. Freeman D. Suspicious minds: The psychology of persecutory delusions. Clin Psychol Rev. 2007;27(4):425-457. doi:10.1016/j.cpr.2006.10.004

4. Freeman D, Startup H, Dunn G, et al. Persecutory delusions and psychological well-being. Soc Psychiatry Psychiatr Epidemiol. 2014;49(7):1045-1050. doi:10.1007/s00127-0130803-y

5. Hor K, Taylor M. Suicide and schizophrenia: a systematic review of rates and risk factors. J Psychopharmacol. 2010;24(4 Suppl):81-90. doi:10.1177/1359786810385490

6. Coid JW, Ullrich S, Kallis $\mathrm{C}$, et al. The relationship between delusions and violence: Findings from the East London first episode psychosis study. JAMA Psychiatry. 2013;70(5):465-471. doi:10.1001/jamapsychiatry.2013.12

7. Freeman D, Bentall RP. The concomitants of conspiracy concerns. Soc Psychiatry Psychiatr Epidemiol. 2017;52(5):595-604. doi:10.1007/S00127-017-1354-4/FIGURES/1

8. Suthaharan $\mathrm{P}$, Reed EJ, Leptourgos $\mathrm{P}$, et al. Paranoia and belief updating during the COVID-19 crisis. Nat Hum Behav 2021 59. 2021;5(9):1190-1202. doi:10.1038/s41562-02101176-8

9. Freeman D, Sheaves B, Goodwin GM, et al. The effects of improving sleep on mental health (OASIS): a randomised controlled trial with mediation analysis. The Lancet Psychiatry. 2017;4(10):749-758. doi:10.1016/S2215-0366(17)30328-0

10. Freeman D, Emsley R, Diamond $R$, et al. Comparison of a theoretically driven cognitive therapy (the Feeling Safe Programme) with befriending for the treatment of persistent persecutory delusions: a parallel, single-blind, randomised controlled trial. The Lancet Psychiatry. 2021;8(8):696-707. doi:10.1016/S2215-0366(21)00158-9

11. Corlett PR, Frith CD, Fletcher PC. From drugs to deprivation: A Bayesian framework for understanding models of psychosis. Psychopharmacology (Berl). 2009;206(4):515-530. doi:10.1007/s00213-009-1561-0

12. Feeney EJ, Groman SM, Taylor JR, Corlett PR. Explaining Delusions: Reducing Uncertainty Through Basic and Computational Neuroscience. Schizophr Bull. 2017;43(2):263-272. doi:10.1093/schbul/sbw194

13. Reed EJ, Uddenberg S, Suthaharan P, et al. Paranoia as a deficit in non-social belief updating. Elife. 2020;9:1-55. doi:10.7554/ELIFE.56345

14. Browning M, Behrens TE, Jocham G, O'Reilly JX, Bishop SJ. Anxious individuals have difficulty learning the causal statistics of aversive environments. Nat Neurosci 2015184. 2015;18(4):590-596. doi:10.1038/nn.3961

15. Hein TP, de Fockert J, Ruiz MH. State anxiety biases estimates of uncertainty and impairs 
reward learning in volatile environments. Neuroimage. 2021;224:117424.

doi:10.1016/J.NEUROIMAGE.2020.117424

16. Freeman D, Bird JC, Loe BS, et al. The Dunn Worry Questionnaire and the Paranoia Worries Questionnaire: new assessments of worry. Psychol Med. 2019. doi:10.1017/S0033291719000588

17. Bebbington $P E, M c B r i d e ~ O$, Steel $C$, et al. The structure of paranoia in the general population. Br J Psychiatry. 2013;202(6):419-427. doi:10.1192/bjp.bp.112.119032

18. Startup H, Freeman D, Garety PA. Persecutory delusions and catastrophic worry in psychosis: Developing the understanding of delusion distress and persistence. Behav Res Ther. 2007;45(3):523-537. doi:10.1016/j.brat.2006.04.006

19. Freeman D, Dunn G, Startup $H$, et al. Effects of cognitive behaviour therapy for worry on persecutory delusions in patients with psychosis (WIT): A parallel, single-blind, randomised controlled trial with a mediation analysis. The Lancet Psychiatry. 2015;2(4):305-313. doi:10.1016/S2215-0366(15)00039-5

20. Moutoussis M, Shahar N, Hauser TU, Dolan RJ. Computation in Psychotherapy, or How Computational Psychiatry Can Aid Learning-Based Psychological Therapies. Comput Psychiatry. 2018;2(0):50-73. doi:10.1162/CPSY_A_00014

21. First MB, Spitzer RL, Gibbon M, Williams JBW. Structured Clinical Intervew for DSM-IV Axis I Disorders- Non-Patient Edition (SCID-I/NP, Version 2.0). (Department BR, ed.). New York; 1995.

22. Kay SR, Fiszbein A, Opler LA. The positive and negative syndrome scale (PANSS) for schizophrenia. Schizophr Bull. 1987;13(2):261-276. http://www.ncbi.nlm.nih.gov/pubmed/3616518.

23. Haddock G, McCarron J, Tarrier N, Faragher EB. Scales to measure dimensions of hallucinations and delusions: The psychotic symptom rating scales (PSYRATS). Psychol Med. 1999;29(4):879-889. doi:10.1017/S0033291799008661

24. Freeman D, Loe BS, Kingdon D, et al. The revised Green et al., Paranoid Thoughts Scale (R-GPTS): psychometric properties, severity ranges, and clinical cut-offs. Psychol Med. 2021;51(2):244-253. doi:10.1017/S0033291719003155

25. Peters E, Joseph S, Day S, Garety P. Measuring Delusional Ideation: The 21-Item Peters et al. Delusions Inventory (PDI). Schizophr Bull. 2004;30(4):1005-1022. doi:10.1093/oxfordjournals.schbul.a007116

26. Fydrich T, Dowdall D, Chambless DL. Reliability and validity of the beck anxiety inventory. J Anxiety Disord. 1992;6(1):55-61. doi:10.1016/0887-6185(92)90026-4

27. Beck, A. T., Steer, R. A., \& Brown G. Beck depression inventory-II. Psychol Assess. 1996.

28. Ehring $T$, Zetsche $U$, Weidacker K, Wahl K, Schönfeld S, Ehlers A. The Perseverative Thinking Questionnaire (PTQ): Validation of a content-independent measure of repetitive negative thinking. J Behav Ther Exp Psychiatry. 2011;42(2):225-232.

doi:10.1016/j.jbtep.2010.12.003

29. Heimberg RG, Horner KJ, Juster HR, et al. Psychometric properties of the Liebowitz Social Anxiety Scale. Psychol Med. 1999;29(1):199-212. doi:10.1017/S0033291798007879

30. Mathys C, Daunizeau J, Friston KJ, Stephan KE. A Bayesian foundation for individual learning under uncertainty. Front Hum Neurosci. 2011;(MAY):9.

doi:10.3389/fnhum.2011.00039 
31. Mathys CD, Lomakina El, Daunizeau J, et al. Uncertainty in perception and the Hierarchical Gaussian Filter. Front Hum Neurosci. 2014;8:825.

doi:10.3389/fnhum.2014.00825

32. Reed, E.J., Uddenberg, S., Mathys, C.D., Taylor, J.R., Groman, S.M., \& Corlett PR. Expecting the unexpected: the paranoid style of belief updating across species. Elife.

33. Deserno L, Boehme R, Mathys C, et al. Volatility Estimates Increase Choice Switching and Relate to Prefrontal Activity in Schizophrenia. Biol Psychiatry Cogn Neurosci Neuroimaging. 2020;5(2):173-183. doi:10.1016/j.bpsc.2019.10.007

34. Waltz JA. The neural underpinnings of cognitive flexibility and their disruption in psychotic illness. Neuroscience. 2017;345:203-217.

doi:10.1016/j.neuroscience.2016.06.005

35. Tervo DGR, Proskurin M, Manakov M, et al. Behavioral Variability through Stochastic Choice and Its Gating by Anterior Cingulate Cortex. Cell. 2014;159(1):21-32.

doi:10.1016/J.CELL.2014.08.037

36. Reddy LF, Waltz JA, Green MF, Wynn JK, Horan WP. Probabilistic Reversal Learning in Schizophrenia: Stability of Deficits and Potential Causal Mechanisms. Schizophr Bull. 2016;42(4):942-951. doi:10.1093/schbul/sbv226

37. Pratt DN, Barch DM, Carter CS, et al. Reliability and Replicability of Implicit and Explicit Reinforcement Learning Paradigms in People With Psychotic Disorders. Schizophr Bull. 2021;47(3):731-739. doi:10.1093/SCHBUL/SBAA165

38. Coltheart M. On the Distinction between Monothematic and Polythematic Delusions. Mind Lang. 2013;28(1):103-112. doi:10.1111/mila.12011

39. Isham L, Griffith L, Boylan A, et al. Understanding, treating, and renaming grandiose delusions: A qualitative study. Psychol Psychother Theory, Res Pract. November 2019:papt.12260. doi:10.1111/papt.12260

40. Kendler KS, Campbell J. Interventionist causal models in psychiatry: Repositioning the mind-body problem. Psychol Med. 2009;39(6):881-887.

doi:10.1017/S0033291708004467

41. Freeman D, Stahl D, McManus S, et al. Insomnia, worry, anxiety and depression as predictors of the occurrence and persistence of paranoid thinking. Soc Psychiatry Psychiatr Epidemiol. 2012;47(8):1195-1203. doi:10.1007/s00127-011-0433-1

42. Startup H, Pugh K, Dunn G, et al. Worry processes in patients with persecutory delusions. Br J Clin Psychol. 2016;55(4):387-400. doi:10.1111/bjc.12109

43. Anglin DM, Ereshefsky S, Klaunig MJ, et al. From Womb to Neighborhood: A Racial Analysis of Social Determinants of Psychosis in the United States. Am J Psychiatry. 2021;178(7):599-610.

doi:10.1176/APPI.AJP.2020.20071091/ASSET/IMAGES/LARGE/APPI.AJP.2020.20071091F 1.JPEG

44. Kühberger A, Fritz A, Scherndl T. Publication Bias in Psychology: A Diagnosis Based on the Correlation between Effect Size and Sample Size. PLoS One. 2014;9(9):e105825. doi:10.1371/JOURNAL.PONE.0105825

45. Dahiya A V., DeLucia E, McDonnell CG, Scarpa A. A systematic review of technological approaches for autism spectrum disorder assessment in children: Implications for the COVID-19 pandemic. Res Dev Disabil. 2021;109:103852. doi:10.1016/J.RIDD.2021.103852 
46. Watt JA, Lane NE, Veroniki AA, et al. Diagnostic accuracy of virtual cognitive assessment and testing: Systematic review and meta-analysis. J Am Geriatr Soc. 2021;69(6):14291440. doi:10.1111/JGS.17190 


\section{Funding}

This work was supported by the Vanderbilt Department of Psychiatry and Behavioral Sciences (JMS) and K23MH126313 (awarded to JMS). The Authors have declared that there are no conflicts of interest in relation to the subject of this study

\section{Acknowledgements}

We would like to thank Kendall Beals and Lauren Hall for the collection of this data. 
Table 1

\begin{tabular}{|c|c|c|c|}
\hline & $\begin{array}{l}\text { HEALTHY } \\
\text { PARTICIPANTS } \\
\text { N=44 }\end{array}$ & $\begin{array}{c}\text { SCHIZOPHRENIA } \\
\text { PARTICIPANTS } \\
\text { N=42 }\end{array}$ & STATISTIC \\
\hline AGE & $30.1(8.0)$ & $27.4(10.2)$ & $t(84)=.1 .83, p=.07$ \\
\hline GENDER (M/F) & $27 / 17$ & $31 / 11$ & $X^{2}=1.5, p=.22$ \\
\hline $\begin{array}{c}\text { RACE } \\
\text { (CAUCASIAN/AFRICAN } \\
\text { AMERICAN/OTHER) }\end{array}$ & $30 / 9 / 5$ & $27 / 12 / 3$ & $X^{2}=1.0, p=.59$ \\
\hline PERSONAL EDUCATION* & $17.1(2.3)$ & $14.6(2.4)$ & $t(84)=5.0, p<.001$ \\
\hline PARENTAL EDUCATION & $14.7(2.3)$ & $14.6(2.8)$ & $t(84)=.24, p=.81$ \\
\hline PREMORBID IQ* & $114(7.7)$ & $105(13.2)$ & $t(84)=4.0, p<.001$ \\
\hline $\begin{array}{l}\text { GREEN PARANOID THOUGHTS } \\
\text { SCALE- B }\end{array}$ & $1.6(2.7)$ & $1.7(3.8)$ & $t(84)=-.04, p=.97$ \\
\hline PANSS POSITIVE & -- & $13.1(4.1)$ & -- \\
\hline PANSS NEGATIVE & -- & $13.8(6.2)$ & -- \\
\hline PANSS GENERAL & -- & $25.5(5.2)$ & -- \\
\hline
\end{tabular}

F=Female; $I Q$ = Intelligence Quotient; $M=$ Male; PANSS = Positive and Negative Syndrome Scale 


\section{Figure Legends}

Figure 1

A) Task image of 3-PRL. B) Schematic of the 3-level hierarchical gaussian filter (HGF) model.

Figure 2

Schizophrenia participants had significantly greater win-shift behavior but did not significantly differ on lose-stay rate or any of the computational parameters.

Figure 3

Both win-shift rate and $\mu_{3}^{0}$ were significantly associated with paranoia.

Figure 4

Indirect effect analysis. A) in the schizophrenia sample and B) across the entire sample 
Figure 1: Task and HGF model

A.
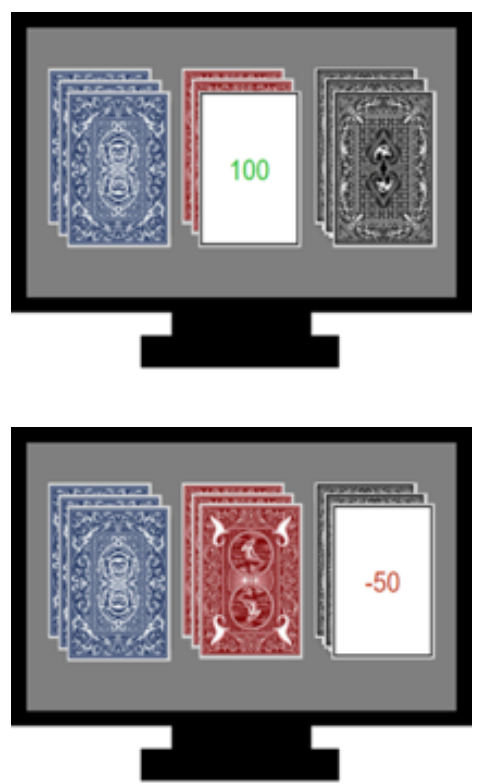

B.

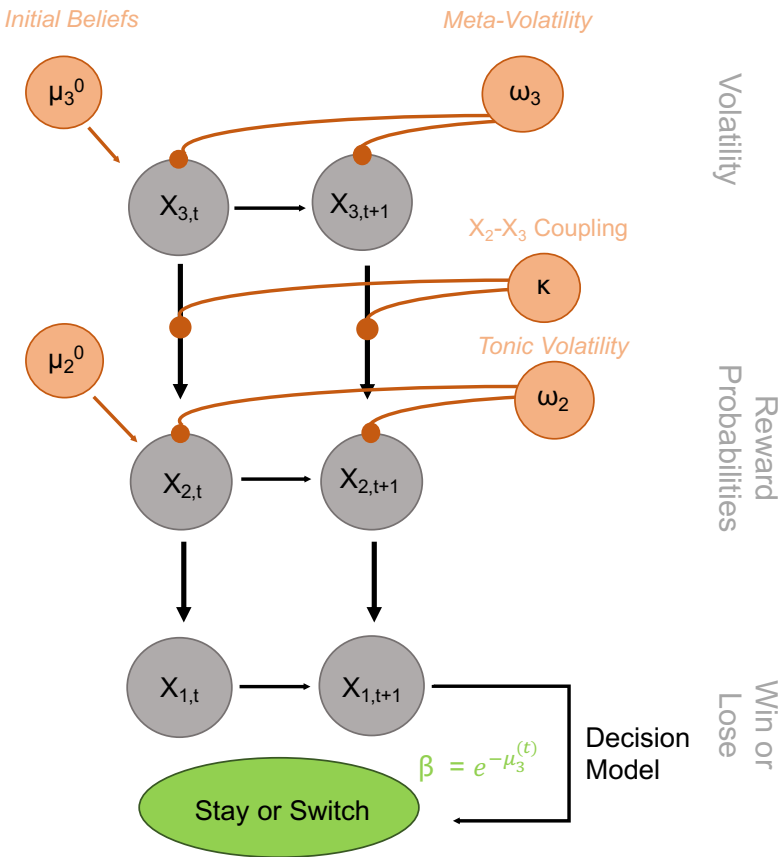

A) Task image of 3-PRL. B) Schematic of the 3-level hierarchical gaussian filter (HGF) model. 
Figure 2: Group differences in belief updating measures

A.

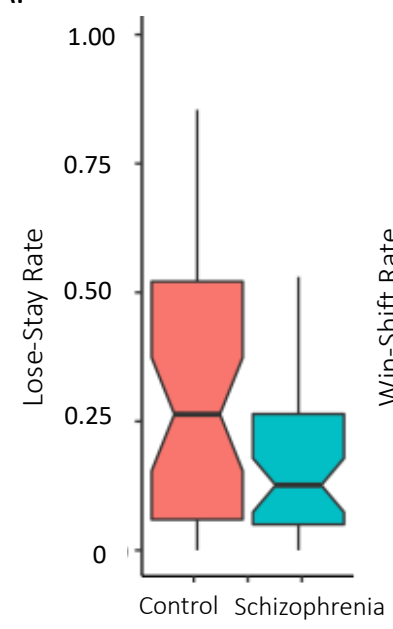

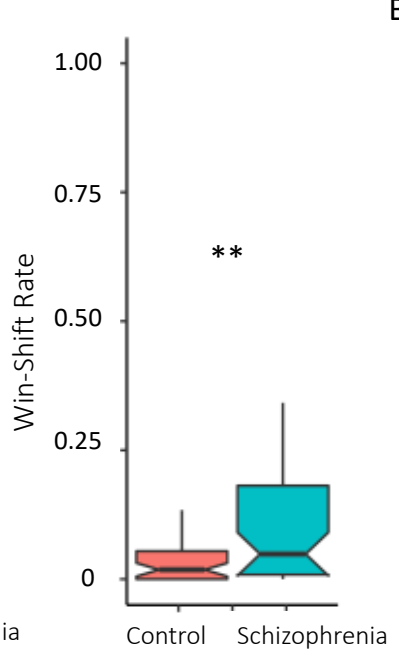

B.
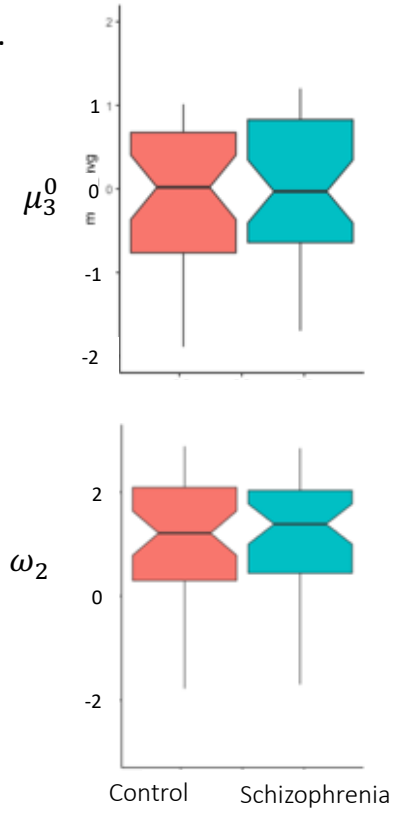
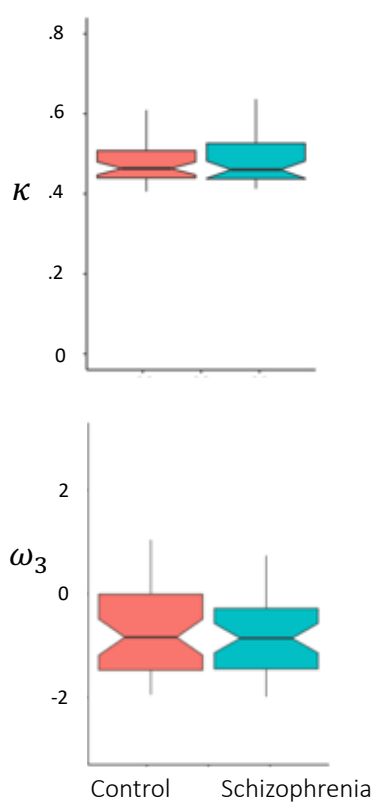

Schizophrenia participants had significantly greater win-shift behavior but did not significantly differ on lose-stay rate or any of the computational parameters. 
Figure 3: Relationships between paranoia and belief updating
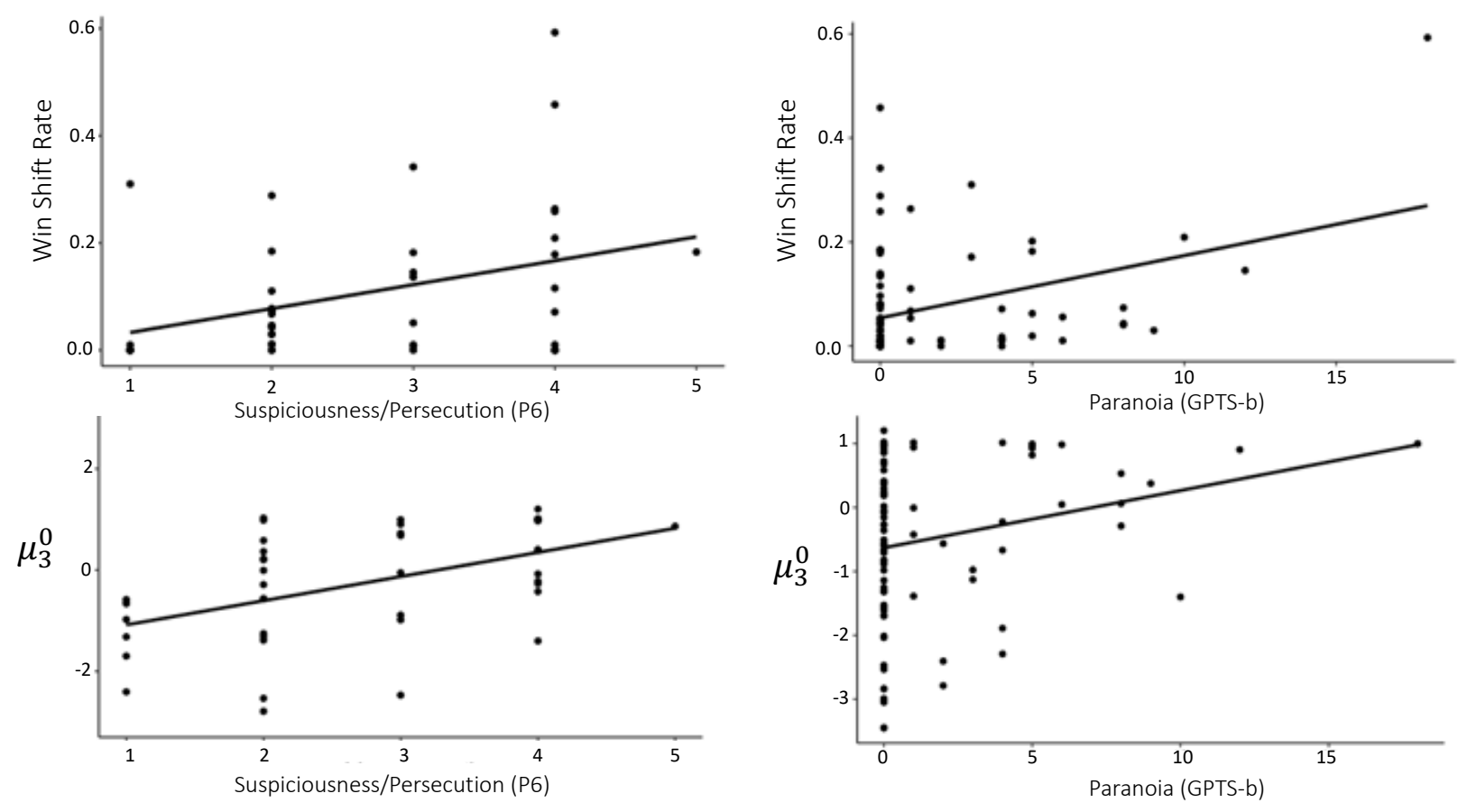

Both win-shift rate and $\mu_{3}^{0}$ were significantly associated with paranoia 
Figure 4: Paranoia shows a significant indirect effect on the relationship between prior on volatility and worry
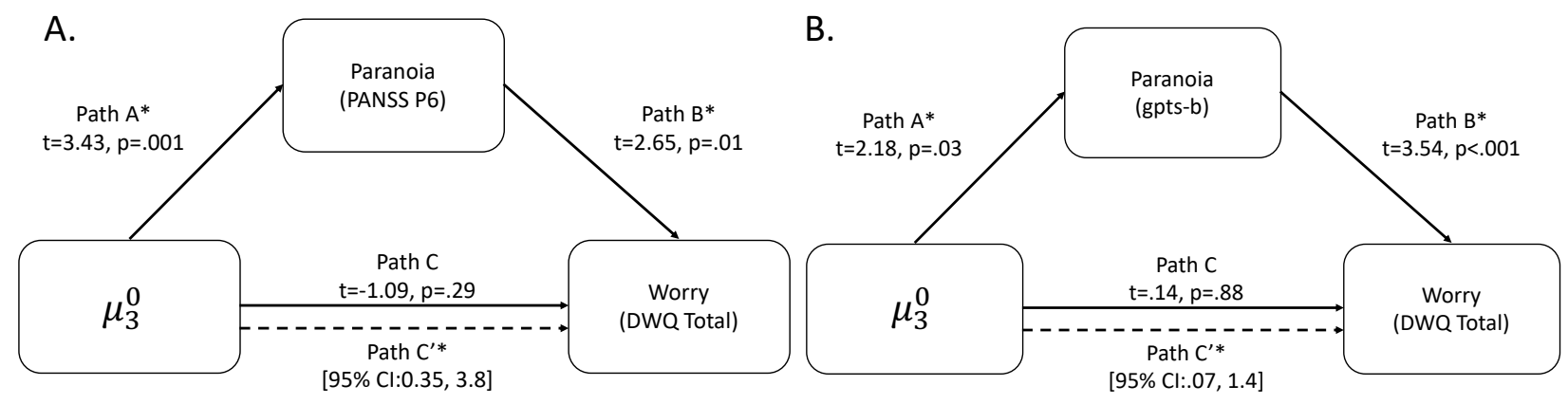

Indirect effect analysis. A) in the schizophrenia sample and B) across the entire sample 


\section{Supplemental Materials}

\section{Results}

\section{Group Differences in Belief Updating: Elevated/Low Paranoia}

Given the lack of a significant group differences in schizophrenia and healthy participants on computational parameters, but a significant association with paranoia, we investigated whether splitting our sample into those with elevated and low paranoia scores (regardless of diagnosis) might reveal differences in belief updating. We used the cutoff score of $\leq 4$ to represent elevated persecutory ideation. Green and colleagues ${ }^{1}$ suggest a cutoff score of 5 for elevated persecutory ideation, however in this exploratory analysis, very few individuals met that definition of elevation ( $N=9$ ). Therefore, we chose a cut-off of $\leq 4$ to gain slightly more power $(\mathrm{N}=13)$ in the between-groups analysis.

Individuals with elevated paranoia had significantly higher $\mu_{3}^{0}(F(1,84)=9.41, p=.003)$ and higher $\kappa(F(1,84)=9.6, p=.003)$ (Figure S1). $\omega_{2}(F(1,84)=3.93, p=.05)$ and $\omega_{3}(F(1,84)=3.1, p=.08)$ did not reach statistical significance. Win-switch behavior was greater in high compared to low paranoia participants $(F(1,84)=4.0, p=.05)$, though did not quite reach statistical significance, and lose-stay behavior $(F(1,84)=.08, p=.78)$ was similar between paranoia groups.

Notably, the healthy and schizophrenia participants in the elevated/low paranoia groups was similar $\left(X^{2}=.66, p=.42\right)$. This suggests that belief updating computational parameters of prior on volatility and unexpected uncertainty are tracking with paranoia, not diagnosis.

\section{Examination of covariates}

Although participants did not significantly differ at the group-level on age, sex, and race, we explored the impact of included these variables as covariates on our primary findings. In addition, we included group as a covariate for relationships with win-shift rate, as this significantly differed between diagnostic groups.

Regarding group differences in behavioral measures, win-shift $(F(1,81)=7.97, p=.006)$ and losestay $(F(1,81)=4.7, p=.03)$ behavior significantly differed between groups with covariates in the model.

In terms of correlations with paranoia, win-shift rate continued to be significantly associated with self-reported paranoia $(r(80)=.29, p=.009)$ and persecutory worry $(r(80)=.29, p=.008)$ across all participants. $\mu_{3}^{0}$ also continued to demonstrate a significant association with interviewer-rated paranoia $(r(37)=.35, p=.03)$; however the relationship was attenuated for selfreported paranoia for both $\mu_{3}^{0}(r(81)=.16, p=.14)$ and $\kappa(r(81)=.13, p=.25)$. 
When examining the high/low paranoia groups, these groups were highly similar on age $(p=.94)$ and sex $(p=.58)$, but had significantly different racial make-ups $(p<.001)$. Specifically, those in the high paranoia group were significantly more likely to be African-American. Although the groups are of very different sizes (73 low paranoia, 13 high paranoia) this racial difference is notable. In fact, when only age and sex are included as covariates, significant associations between paranoia, $\mu_{3}^{0}$ and $\kappa$ are observed for both self-reported paranoia ( $\left.p^{\prime} s<.04\right)$ and interview-rated paranoia ( $\left.p^{\prime} s<.011\right)$.

That said, the high paranoia group continued to demonstrate significantly greater $\mu_{3}^{0}$, when controlling for age, sex and race $(F(1,81)=5.36, p=.02)$ and group differences in $\kappa$ were trending $(F(1,81)=3.62, p=.06)$. This suggests that race is a potentially important demographic factor for understanding the relationship between belief updating parameters and paranoia, but that it does not fully explain the elevated prior on volatility, unexpected uncertainty, and winswitching observed in relationship to higher paranoia.

1. Freeman D, Loe BS, Kingdon D, et al. The revised Green et al., Paranoid Thoughts Scale (R-GPTS): psychometric properties, severity ranges, and clinical cut-offs. Psychol Med. 2021;51(2):244-253. doi:10.1017/S0033291719003155 
Figure S1: Differences in belief updating computational parameters based on paranoia group
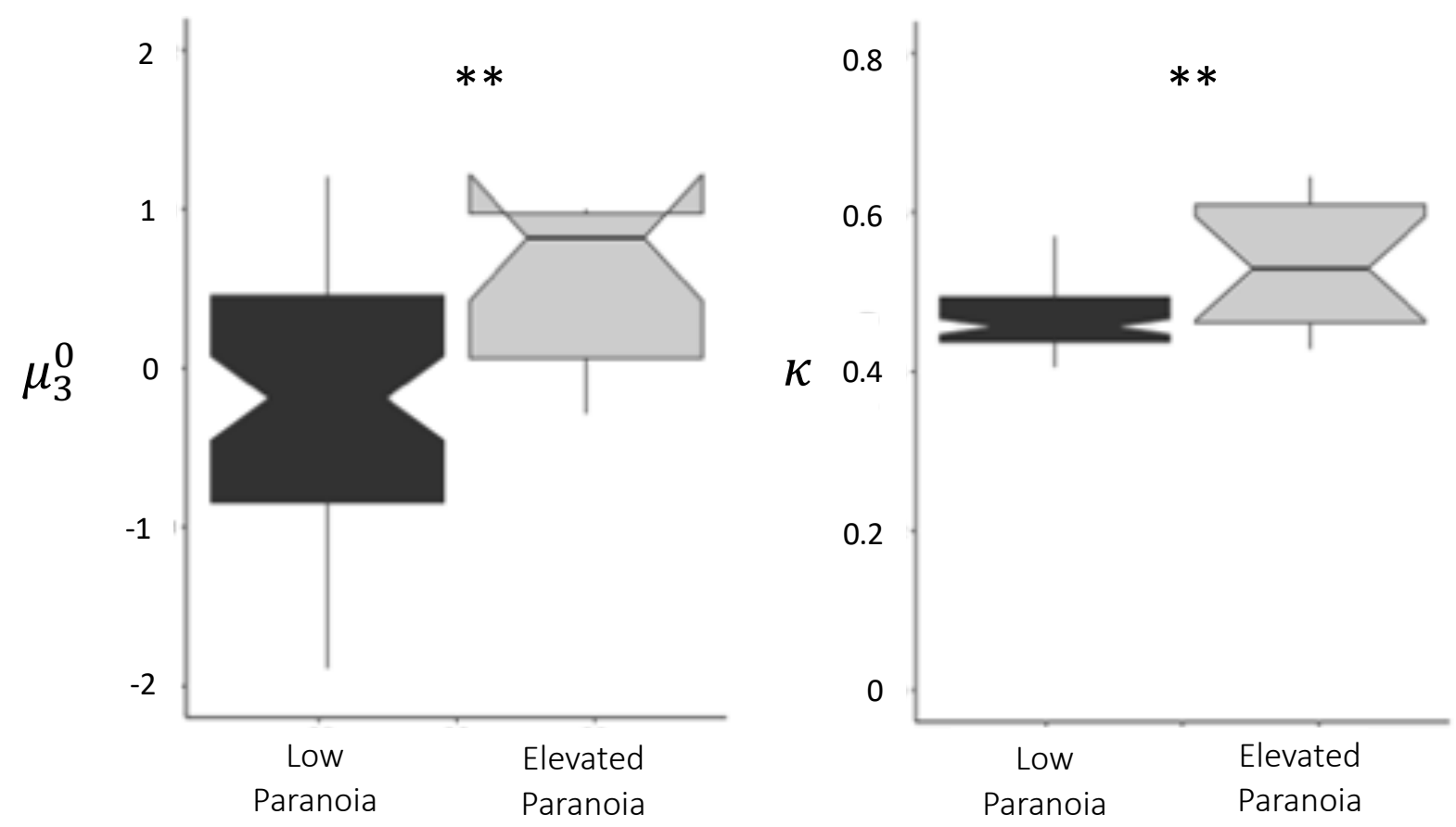

Group differences in $\mu_{3}^{0}$ and $\kappa$ for individuals with low paranoia (R-GPTS scores $\leq 4$ ) and elevated paranoia (R-GPTS 5+)27. The elevated paranoia group demonstrated higher $\mu_{3}^{0}$ and $\kappa$. 
